\title{
Effekte der digitalen Transformation des Kranken- hauses auf den Wandel des Berufsbildes Arzt
}

\author{
David Matusiewicz, Jana Aulenkamp und Jochen A. Werner \\ ○ Der/die Autor(en) 2019 \\ J. Klauber et al. (Hrsg.), Krankenhaus-Report 2019 \\ https://doi.org/10.1007/978-3-662-58225-1_8
}

\section{Zusammenfassung}

Die digitale Transformation des Krankenhauses als „smart factory“ stellt die Organisation im Allgemeinen und das Berufsbild des Arztes im Besonderen vor enorme Veränderungen. Dies geht mit einer veränderten medizinischen Berufsausbildung einher und ist mit neuen beruflichen Anforderungen verbunden. Neben den neuen Möglichkeiten in der Diagnostik und Therapie fallen einerseits bisherige Aufgaben weg, während andererseits neue Aufgaben hinzukommen. Auch die Arzt-Patienten-Beziehung verändert sich, da der Arzt einem zunehmend gut informierten Patienten gegenübersteht. Der Beitrag diskutiert thesenhaft, wie sich die Berufsausübung des Arztes im Krankenhaus durch die verstärkte Einführung technischer Lösungen verändert, was dies in Folge für die berufliche Ausbildung bedeutet, welche neuen Anforderungen (etwa Spezialisierungen) für die Ärzte hiermit verbunden sind und wie sich schließlich hierdurch das Verhältnis zu anderen Berufsgruppen und zum Patienten im Krankenhaus wandelt.

The digital transformation of the hospital as a "smart factory" represents enormous changes for the organization in general and the profession of the physician in particular. This is associated with a change in medical education and with new professional requirements. In addition to the new possibilities in diagnostics and therapy, previous tasks are no longer necessary, while new tasks are added. The physician-patient relationship is also changing as the physician faces an increasingly well-informed patient who regards him as a service provider. The paper discusses how the routines of the hospital physician is changing due to the increased introduction of technical solutions, what this means for vocational training, which new requirements (such as specialisations) for physicians are involved and how, finally, the relationship with other professional groups and with patients in hospital is changing as a result.

\subsection{Hintergrund und Einführung}

Der sozio-demografische Wandel, die immer kürzer werdenden Abstände der Gesundheitsreformen, die Ökonomisierung der Medizin, der Fachkräfte- mangel und auch der medizinisch-technische Fortschritt führen dazu, dass das Thema Wandel im „Ökosystem“ Krankenhaus zum Normalfall geworden ist (vgl. ausführlich Matusiewicz et al. 2017). Das Krankenhaus befindet sich heute in einer dyna- 
mischen Umwelt, in der die digitale Transformation eine entscheidende Rolle spielt.

Allerdings werden die digitalen Möglichkeiten innerhalb des Gesundheitswesens im Vergleich $\mathrm{zu}$ anderen Branchen bislang noch eher selten und längst nicht flächendeckend eingesetzt (vgl. Henn 2015; Liebrich 2017). Beispielsweise liegt der Grad der Kollaboration (Branchen-Reifegrad) des Gesundheitswesens nach einer Studie von Terra Consulting Partners (TCP) noch schätzungsweise 10 bis 15 Jahre hinter anderen Branchen wie dem Handel oder der Automobilbranche zurück (KadeLamprecht und Sander 2017). Doch die Gesundheitsbranche hat in den letzten Jahren verstanden, dass Kommunikationswege mithilfe von unstrukturierten handschriftlichen Patientenakten und Fax-Geräten nicht mehr State of the Art sind und versucht sich seitdem zeitnah neu zu erfinden. Dies nicht zuletzt, weil der Patient und seine Angehörigen dies einfordern. Zudem drängen neue Akteure auf den Markt wie die GAFA (Google, Amazon, Facebook und Apple), die ebenso neue starke Impulse setzen und die Branche derzeit sogar punktuell vor sich hertreiben.

All dies bringt ebenso enorme Chancen mit sich. Denn im Gesundheitswesen gibt es Effizienzreserven zu heben. Hierzu zählen bspw. Schnittstellenprobleme, eine fehlende Customer Centricity (neudeutsch für „Patient als Mittelpunkt“), Kommunikationsdefizite und generelle Steuerungsprobleme. Es kann darüber diskutiert werden, ob die Berufsgruppen heute - wie es in der modernen Managementlehre vorzufinden ist - gemeinsam im Team zusammenarbeiten. Befürworter argumentieren, dass OP-Teams, Funktionsabteilungen, Stationsteams und die Verwaltung bereits zusammenarbeiten. Aus berufspolitischer Sicht halten Kritiker dagegen, dass die Gesundheitsberufe aus Tradition eher in einem „Kastensystem“ nebeneinander arbeiten - hierzu gibt es allerdings lediglich anekdotenhafte Evidenz. Laut einer Umfrage des Marburger Bundes verbringen 33 Prozent der befragten Ärzte durchschnittlich zwei Stunden pro Tag mit Dokumentation und der Erfassung von Daten. 29 Prozent gaben an, dass der tägliche Aufwand bei zwei bis drei Stunden liegt und 26 Prozent sogar bei mehr als drei Stunden (vgl. Marburger Bund 2017). Auf der einen Seite sollen die Ärzte dem Patienten genug
Zeit widmen, während sie auf der anderen Seite mit einem riesigen Aufwand an nicht-ärztlichen Leistungen wie etwa einer zunehmenden Bürokratisierung im beruflichen Alltag konfrontiert werden. Durch den sinnvollen und flächendeckenden Einsatz digitaler Technologien im Krankenhauswesen könnten solche Tätigkeiten des Arztes künftig wegfallen oder zumindest reduziert oder automatisiert werden. Dies kann für den Einzelnen eine Entlastung und Unterstützung der gestiegenen Anforderungen im Klinikalltag bedeuten und zukünftig die Qualität der medizinischen Gesundheitsversorgung im Krankenhaus verbessern. Laut einer Studie des Digitalverbands Bitkom, die gemeinsam mit dem Ärzteverband Hartmannbund durchgeführt und bei der 477 niedergelassene Ärzte und Krankenhausärzte aller Fachrichtungen und Funktionen zum Thema Digitalisierung befragt wurden, besteht innerhalb der Ärzteschaft grundsätzlich Offenheit gegenüber dem Einsatz von digitalen Lösungen und Technologie im Krankenhaus. Die bisherige $\mathrm{Zu}-$ rückhaltung beim Ausbau einer digitalen Infrastruktur in deutschen Krankenhäusern ist vor allem auf die strengen Datenschutzvorgaben und Richtlinien in Bezug auf die IT-Sicherheit zurückzuführen (vgl. Bitkom Research 2017). Der sinnvolle und sichere Einsatz der Digitalisierung im Gesundheitswesen geht mit der Chance einher, Ärzte in ihrem beruflichen Alltag zu entlasten und ihnen unterstützend zur Seite zu stehen. Dadurch kann die medizinische Versorgungsqualität verbessert und die Krankenhausprozesse können effizienter gestalten werden. Die enormen Potenziale werden im deutschen Gesundheitswesen zunehmend Berücksichtigung finden (vgl. PWC 2016).

Es stellt sich die Frage, welchen Effekt die Digitalisierung auf das Berufsbild des Arztes in Zukunft haben wird. Die Digitalisierung könnte hierbei in Rationalisierungspotenziale (sicherer, schneller, vielfältiger kommunizieren und dokumentieren), Mehrwertpotenziale (neue medizinische Möglichkeiten schaffen, etwa durch neue Bildgebung oder Laborarrays, vernetzte Kooperation oder Versorgungsforschung) und die Entstehung von künstlicher Intelligenz mit dem Potenzial, ärztliche Arbeit ihrem Wesen nach zu verändern (z. B. Monitoringund Alarmsysteme), differenziert werden. Im vorliegenden Artikel werden insbesondere die Berufs- 
ausbildung, Berufsausübung, Spezialisierung der Berufe, das Verhältnis der Berufsgruppen zueinander und die Arzt-Patienten-Beziehung näher betrachtet. Der Einsatz der Digitalisierung im Bereich der Administration, Materialwirtschaft und Qualitätsprüfung wird im vorliegenden Artikel nur nachrangig betrachtet.

\subsection{Berufsausbildung}

Schon heute ist abzusehen, dass Ärzte mit umfangreichen Daten konfrontiert werden, die bei der Diagnose und Behandlung berücksichtigt und analysiert werden müssen. Infolgedessen ist es notwendig, entsprechende Inhalte zum Umgang mit Daten, zu deren Erhebung und der klinischen Entscheidungsfindung auf Basis dieser Ergebnisse in die Curricula der medizinischen Fakultäten zu integrieren. Dabei gilt, dass die Sicherheit des Patienten und die Handlungsfreiheit des Arztes stets im Vordergrund stehen (vgl. Krüger 2018). Infolgedessen ist heute schon absehbar, dass der Wandel des Berufsbildes von Ärzten mit neuen beruflichen Anforderungen an die medizinische Ausbildung in Studium und Weiterbildung einhergeht. Vor diesem Hintergrund wird für die erfolgreiche Umsetzung der digitalen Transformation eine begleitende Ausbildung erforderlich sein. Eine Herausforderung ist, dass die Ausbildung den Grundstein für eine kontinuierliche professionelle Entwicklung im Umgang mit Daten und Technologien legen muss, die es heute noch nicht ausreichend gibt. Der Paradigmenwechsel in der Ausbildung muss also den bisherigen schlichten Positivismus (Faktenzentrierung, Multiple-Choice-Prüfungen) durch eine methodenzentrierte und wissenschaftsorientierte Haltung und Kompetenzentwicklung ersetzen. Das ist die eigentliche Herausforderung für die Curriculumsentwicklung, vor der die fächerorientierten Fakultäten stehen.

Die Ausbildung sollte den Nachwuchs auf die kommenden Veränderungen vorbereiten. Neue Medien und Technologien halten Einzug in unser Gesundheitswesen, die Aufgabenverteilung zwischen den Berufsgruppen wird überdacht und neue Versorgungsformen werden erprobt. Vor allem neue technische Lösungen eröffnen Möglichkeiten für neue Konzepte in Kliniken. Andererseits erhalten die Patienten einen einfacheren Zugang zu Wissen und Informationen, um mehr über ihre Gesundheit zu erfahren. Dem Umgang mit diesen Entwicklungen sollte bereits im Studium Raum gegeben werden, um einen reflektierten Umgang mit ihnen zu erlernen. Die medizinische Ausbildung befindet sich ebenso wie das gesamte Gesundheitswesen im Wandel. Angestoßen wird dies unter anderem durch den Masterplan „Medizinstudium 2020“ mit dem Bestreben, das Studium neu zu strukturieren, praxisorientierter zu gestalten und die hausärztliche Versorgung sicherzustellen. Der Wissenserwerb soll Teil eines kompetenzorientierten Studienganges sein, dessen Ziel es ist, Wissens- und Handlungskompetenzen zielgerichtet auf ein Absolventenprofil am Ende des Studiums zu vermitteln. Bereits in der ÄAppO ist in $\$ 1$ Abs. 1 definiert:

"Ziel der ärztlichen Ausbildung ist der wissenschaftlich und praktisch in der Medizin ausgebildete Arzt, der zur eigenverantwortlichen und selbstständigen ärztlichen Berufsausübung, zur Weiterbildung und zu ständiger Fortbildung befähigt ist. Die Ausbildung soll grundlegende Kenntnisse, Fähigkeiten und Fertigkeiten in allen Fächern vermitteln, die für eine umfassende Gesundheitsversorgung der Bevölkerung erforderlich sind. Die medizinische Ausbildung zum Arzt wird auf wissenschaftlicher Grundlage praxisund patientenbezogen durchgeführt."

Diese Zielbestimmung besteht schon sehr lange und hat offensichtlich bisher nicht hingereicht, Kompetenzorientierung und aktive Wissenschaftskompetenz sicherzustellen. Das Medizinstudium muss Ärzte auf verschiedenste Bereiche und Rollen, die diese später einnehmen werden, vorbereiten. Neben wissenschaftlichen Kompetenzen, Faktenwissen über biologische, (patho-)physiologische oder psychosoziale Aspekte sind Kompetenzen zur Interaktion, Teamarbeit sowie die Anwendung von Verfahren ebenso hoch zu gewichten. Des Weiteren sollte die medizinische Ausbildung Raum und Zeit für die Selbstreflexion der auszubildenden Studierenden beinhalten (MFT 2015).

Parallel mit der inhaltlichen Neuausrichtung des Medizinstudiums sorgt auch die Umsetzung/ Implementierung digitaler Strukturen in die medizinische Versorgung für eine Reihe von Herausforderungen an die Ausbildung: 
- Fehlende politische Betonung von „Digitalisierung" in der ärztlichen Ausbildung

- Neue Anforderungen an die Qualifizierung des Lehrpersonals

- Grundlegende Einbettung digitaler Strukturen in die Arbeits- und Organisationsstrukturen

- Hoher finanzieller Aufwand

Digitale Transformationsprozesse in der Lehre finden bisher keine adäquate Beachtung im „Masterplan Medizinstudium 2020“ oder im „Nationalen kompetenzorientierten Lernzielkatalog Medizin“ (NKLM) des Medizinischen Fakultätentages (MFT) (BMBF 2017; MFT 2015). Im Koalitionsvertrag 2018 wird generell auf die Digitalisierung der Hochschulen verwiesen, jedoch im Hinblick auf das Medizinstudium lediglich auf eine schnelle Umsetzung des „Masterplans Medizinstudium 2020“ gedrängt, anstatt die Digitalisierung und deren Auswirkungen auf die Berufe und deren Arbeitsweise im Krankenhaus mit zu beleuchten. Diesen Versäumnissen entsprechend fühlen sich viele der praktizierenden Ärzte auf Teile der Digitalisierung wie z. B. das Telemonitoring nicht ausreichend vorbereitet oder es fehlen in vielen Bereichen Fortbildungen für jetzige praktizierende Ärztinnen und Ärzte, um sich mit diesem Themenkomplex ausreichend vertraut $\mathrm{zu}$ machen (Altpeter 2017; Kuhn 2018).

Herausfordernd ist ebenfalls die Einbettung neuer Technologien sowie der Digitalisierung in die Arbeitsorganisation und die Arbeitsstrukturen. Eines der grundlegenden Elemente für die Einführung digitaler Lehrkonzepte ist die grundsätzliche digitale Infrastruktur von Krankenhäusern. Kurz gesagt: Das Faxgerät sollte nicht die modernste digitale Errungenschaft einer Klinik sein, um Studierenden zu vermitteln, wie man Telemedizin betreibt. Die Uniklinik bzw. das Lehrkrankenhaus können allerdings nur die Arbeitsumgebung nutzen und dem Nachwuchs vorstellen, die zur Verfügung steht. Ein weiterer denkbarer Lösungsansatz wäre es, den Nachwuchs in die Entwicklung des (technischen, organisatorischen) Fortschritts aktiv einzubeziehen und so das Potenzial der jungen Generation zu nutzen. Damit ist verbunden, dass qualifizierende Strukturen geschaffen werden und sich das Lehrpersonal den Anforderungen entsprechend neu qualifizieren muss. Eine reine Aktualisierung des fachspe- zifischen Wissens reicht hierfür nicht aus, sondern es müssen neue „digitale Kompetenzen“ erlernt werden (Becka et al. 2017). Dies erfordert eine fächerübergreifende Zusammenarbeit, da für alle Berufsgruppen dieser Themenbereich Veränderungen und neue Chancen mit sich bringt (Feldmann und Wolff 2018). Auf der anderen Seite bietet die gegenwärtige Unvollkommenheit viel Gestaltungsfreiraum und Möglichkeiten für Pionierarbeit.

Die Ausbildung von Medizinstudierenden ist durch die Digitalisierung und die neuen Technologien gleich zweifach betroffen. Durch die „Digitalisierung der Lehre" auf der einen Seite finden immer mehr neue Lehr- und Lernformate (z. B. Online-Vorlesungen oder eLearning-Plattformen) Einzug in das Studium. Dabei ist die digitale Transformation der Lehre nicht technologiegetriebener Selbstzweck, sondern Mittel für ein neues Absolventenprofil. Auf der anderen Seite ist durch die „Digitalisierung als Lehrinhalt" das Thema Digitalisierung des Gesundheitswesens oder digitale Transformation präsent. Die Möglichkeiten sind extrem vielfältig: von Simulationen mit App-basierten Konzepten über Telemedizin bis hin zu Diskussion über ethische und rechtliche Fragestellungen oder dem Bauen von Zukunftsszenarien zu einer neuausgerichteten Zusammenarbeit im Krankenhaus. Trotz schleppender Umsetzung digitaler Entwicklungen an den Hochschulen, konnten daher einige innovative Konzepte entstehen. Ein Beispiel ist die Universität in Mainz, die in einem Pilotprojekt ebenfalls die Digitalisierung der Medizin in den Lehrplan aufgenommen hat und diese Einführung evaluiert (vgl. Kuhn et al. 2018). Darüber hinaus gibt es in Göttingen eine virtuelle Notaufnahme, die komplexe Handlungsschritte in Notfallsituationen trainiert. In Aachen findet sich ein Blended-LearningKonzept zum Kurs Propädeutik der Organsysteme. An der Universität Witten/Herdecke ist die digitale Versorgung zentraler Bestandteil der Ausbildung. Darüber hinaus finden sich derzeit noch weitere Beispiele, die gesammelt in - Tab. 8.1 aufgeführt sind.

Das Angebot an digitalen Medien oder neuen Konzepten für die Lehre ist enorm groß. Neben eLearning-Plattformen über digitale Prüfungen zu Simulationen mit VR-Brillen werden noch viele weitere Formate angeboten. Hierfür exemplarisch ist das Curriculum an der medizinischen Fakultät Essen. Medien für Lehr- und Lernformate werden 
Tab. 8.1 Beispiele digitaler Transformation im Medizinstudium nach verschiedenen Standorten

\section{Digitale Transformation der Lehre}

\section{Georg-August-Universität Göttingen:}

Emerge - Virtuelle Notaufnahme

Komplexe Handlungsschritte in Notfallsituationen werden standardisiert in einem Software-Programm trainiert

- Virtuelles Training von Akut- und Stresssituationen

- Debriefing, Feedback und Reflexion der Szenarien

Durchführung im 4. und 5. Studiensemester

\section{Rheinisch-Westfälische Technische Hochschule Aachen:}

Blended-Learning-Konzept zum Kurs Propädeutik der Organsysteme

Im Rahmen des organzentrierten Systemblocks Nervensystem und in Zusammenarbeit mit dem Audiovisuellen Medienzentrum der Medizinischen Fakultät der RWTH Aachen (AVMZ) wird eine Lernanwendung für die anatomischen Inhalte des Systemblocks eingesetzt.

\section{Universität Duisburg-Essen:}

"Echokardiographie Basic Augmented Reality"

Wahlfach an zwei Terminen:

- Diagnose von Herzpathologien anhand des Echo-Simu-

latormodells lernen

\section{Ruprecht-Karls-Universität Heidelberg:}

Studentischer kompetenzorientierter Progresstest

Freiwillige ePrüfung für Überprüfung des Lernfortschritts:

- 120 MC-Fragen inkl. Video-Sequenzen (umfassen

Themengebiete aus allen Semesterstufen $\rightarrow$ Kompetenzbereichen und Fächergruppen zugeordnet)

- 10 "Situational-Judgement-Test"-Fragen (SJT), mit Fokus auf professionellem Handeln

Bundesweit für Medizinstudierende von 17 Fakultäten verfügbar

\section{Digitale Transformation als Lerninhalt}

\section{Johannes-Gutenberg-Universität Mainz:}

Curriculum 4.0 - Medizin im digitalen Zeitalter

5 Module + Diskussionsrunden + Zukunftsszenarien

- Digitale Arzt-Patienten-Kommunikation

- Smart Devices und Medizinische Apps

- Telemedizin

- Virtual Reality, Augmented Reality und computerassistierte Chirurgie

- Individualisierte Medizin und Big Data

Modul E-Learning-Einheit und dreistündige Präsenzunterrichtseinheit

\section{Universität Witten/Herdecke:}

Quo vadis Medizinstudium? Digitale Versorgung als zentraler Bestandteil der Ausbildung

- Multiprofessionell

- Themen: Big Data über Datensicherheit bis hin zur Startup-Gründung

- Erfahrungsbasiertes Studium

- An dem Kurs kann nicht nur in Präsenz, sondern auch live online via Adobe Connect teilgenommen werden

Integriert in Studium fundamentale

\section{Ruhr-Universität Bochum:}

"Medizin 4.0 - Das Betriebssystem der digitalen Medizin"

Wahlfach über ein Wochenende zu den Themen:

- Erstellung von Digitalisierungskonzepten

- Agiles Management/Prozessmanagement

- Fehlerkultur

Klinik

\section{Eberhard-Karls-Universität Tübingen:}

"iTüpFerl" - in Tübingen psychosoziale Fertigkeiten erlernen

Vorlesung - Praktikum - Seminar/Längsschnittcurriculum:

- Arzt-Patienten-Kommunikation und Teamarbeit

- Einflüsse des digital informierten Patienten auf die Kommunikation

- Online-Schulungsvideos und praktische Übungen

Vorklinik und Klinik etwa in die folgenden Kategorien aufgeteilt (Persike und Friedrich 2016):

Klassisch: Klassische digitale Medien und Kommunikationstools

- Digitale Präsentationstools

- E-Mail

- Fachspezifische Datenbanken

- Digitale Texte
Sozial: Soziale Kommunikationstools

- Blogs

- Chats

- Foren

- Microblogging

- Soziale Netzwerke 
E-Examen: Elektronische Prüfungssysteme

- E-Assessment

- E-Prüfung

- Electronic Objective Structured Clinical Exam (E-OSCE)

Medien: Audio-/videobasierte Medien

- Audio

- Video

- Tutorials (Podcasts, Screencasts)

Interaktiv: Interaktive Tools und Formate

- Lernspiele und Unterhaltungsspiele

- Simulationen

- Webkonferenzen

- Wikis

- Interaktive fachspezifische Werkzeuge (z. B. virtuelle Operationen)

- Online-Office-Tools

eLearning bezeichnet den Einsatz elektronischer Medien in der Wissensvermittlung, sofern diese dem Lernenden einen Mehrwert bieten (Boeker und Klar 2006). Blended Learning verbindet Präsenz- und Onlinelernformate. Dadurch wird eine größere Flexibilität für die Lernatmosphäre geschaffen und das eigenverantwortliche Lernen erleichtert. Zusätzlich konnten positive Effekte bezüglich des erworbenen deklarativen und prozeduralen Wissens im Vergleich $\mathrm{zu}$ reinen E-Learning-Settings sowie gegenüber traditionellen Lernszenarien nachgewiesen werden (Kuhn et al. 2018). Das Inverted-Classroom-Konzept ist als eine Erweiterung des Blended Learning zu verstehen und beinhaltet, dass vor der Präsenzveranstaltung eine eigenverantwortliche eLearning-Einheit absolviert wird. So kann bereits in der Präsenzveranstaltung auf die gelernten Inhalte zurückgegriffen werden. Diese Methode fördert die Motivation und Lernzufriedenheit der Studierenden und führt zu besseren Lernergebnissen (Lage et al. 2000). Mittlerweile findet sich dieses Konzept an vielen Universitäten. So werden zum Beispiel an der Ruhr-Universität Bochum die Studierenden im Rahmen des Anästhesie- und Notfallunterrichts im Vorhinein durch einen Online-Kurs auf die Gruppensimulation der Narkoseeinleitung und Notfallsimulationen vorbereitet.

In der medizinischen Ausbildung werden verstärkt Simulationen vor allem im Bereich der Anäs- thesie, Notfallmedizin oder Chirurgie eingesetzt. Oft wird dabei den Studierenden ermöglicht, ein in der Klinik alltäglich auftretendes Problem zu lösen (z. B. Blutdruckabfall während der Narkoseeinleitung an einer technikgesteuerten Puppe). Der Einsatz von Simulationen wirkt sich vor allem positiv auf das Erlernen von Fertigkeiten und das „clinical reasoning", also das wissenschaftliche Denken aus (Stegmann und Fischer 2016). Des Weiteren wird der Einsatz von virtuellen Patientinnen und Patienten verstärkt genutzt. Dies kann etliche Vorteile mit sich bringen, da es in der Lehre schwierig ist, in kurzer Zeit alle möglichen Krankheitsbilder zu zeigen. Auch wenn virtuelle Patienten in der Lehre helfen können, darf der finanzielle Aufwand, diese „Patienten" zu generieren, nicht in Vergessenheit geraten (Ellaway et al. 2008). In einer Studie, die die Studierendenperspektive auf die neuen Formate untersucht hat, wurde ebenfalls deutlich, dass die Nutzung digitaler Medien sehr standortspezifisch ist. Digitale Lern- und Lehrmedien sind nicht per se qualifizierte Lehrformate, sondern dann sinnvoll, wenn sie in ein globales, evidenzbasiertes Lernkonzept integriert sind (Persike und Friedrich 2016).

Neben der Digitalisierung der Lehre an den Medizinischen Hochschulen gibt es auch bereits Online-Angebote von Drittanbietern. So bieten sich unter anderem sas Lernsystem AMBOSS der Firma Miamed oder die „via medici“-Plattform des Thieme-Verlages zum Selbstlernstudium an. Die Angebote können auf die Examina der Medizinstudierenden angepasst werden; das medizinische Wissen ist sowohl online als auch offline abrufbar. Durch Probefragen, Kommentarfunktionen oder Erklärvideos ergänzen diese Plattformen ihr Angebot und bieten somit mehr Vielfalt als das herkömmliche Lernen aus Büchern.

\section{Data Literacy im Medizinstudium}

Ein zentraler Bestandteil der digitalen Transformation als Lerninhalt ist das Thema Data Literacy. Dies umfasst die „Fähigkeit, planvoll mit Daten umzugehen und sie im jeweiligen Kontext bewusst einsetzen und hinterfragen zu können. Dies beinhaltet die Fähigkeit, Daten zu erfassen, erkunden, managen, kuratieren, analysieren, visualisieren, interpretieren, kontextualisieren, beurteilen und anzuwenden" (Michel 2018). Der Begriff Health Literacy wird in Deutschland aus Praktikabilitätsgründen mit "Gesundheitskompetenz" (anstelle von "gesundheitsbezogene Literalität") übersetzt. Seit Anfang des Jahres 2018 hat Deutschland nach dem Vorbild 
anderer Länder einen „Nationalen Aktionsplan zur Stärkung der Gesundheitskompetenz" erstellt (vgl. ausführlich Schaeffer et al. 2018). Denn ein kompetenter Umgang mit Daten sowie deren kritische und adäquate Interpretation sind wichtig. Gerade im Zeitalter von Big Data wird es immer essentieller, dass zukünftige Ärztinnen und Ärzte in kurzer Zeit viele Datenmengen analysieren und erklären können (Kuhn et al. 2018). Zum jetzigen Zeitpunkt wird dieser Berufsgruppe noch viel Vertrauen entgegengebracht, wobei einige Patienten dazu neigen, sich wohler zu fühlen, wenn zum Beispiel bei der Diagnose digitale Unterstützung gegeben ist (Lang 2017; Nelles 2017). Das könnte sich ändern, wenn erkennbar wird, welche Defizite die Berufsausbildung im Umgang mit Daten hat. Gerd Gigerenzer vom Max-Planck-Institut für Bildungsforschung erhebt öfters den Befund, dass Ärzte schon mit einfachen Informationen zur Testvalidität (bedingte Wahrscheinlichkeiten - „Bayes-Theorem") nicht umgehen können (vgl. Keller et al. 2018). An der Stelle stellt sich aber auch die Frage, wie weit EntscheidungsunterstützungsSysteme für die tägliche Routine sinnvoll sind. Der Wissenschaftsrat hat bereits 2014 eine Empfehlung zur "Weiterentwicklung des Medizinstudiums in Deutschland auf Grundlage einer Bestandsaufnahme der humanmedizinischen Modellstudiengänge" vorgelegt und Lösungsansätze wie unter anderem zwei wissenschaftliche Arbeiten für Medizinstudierende unterbreitet (vgl. Wissenschaftsrat 2014). Diesen Veränderungen muss in der medizinischen Ausbildung Rechnung getragen werden, damit neben den kommunikativen Fähigkeiten für die Interaktion mit dem Patienten auch fundiertes Wissen vorhanden ist. Ein transdisziplinärer Ansatz unter Einbeziehung vieler verschiedener Experten kann hier in der Lehre hilfreich sein (Kuhn et al. 2018). Die Integration von Data Literacy in das Medizinstudium ist für die Neudefinition des professionellen Rollenverständnisses, der Kompetenzorientierung sowie des interdisziplinären und multiprofessionellen Arbeitens von großer Bedeutung (Kuhn et al. 2017). Dies wird als eine der bedeutsamsten Fähigkeiten im Rahmen der Digitalisierung des Gesundheitssystems erachtet, bleibt bislang jedoch sowohl in den bestehenden Lehrplänen des Medizinstudiums als auch in aktuellen Reformen weitestgehend unberücksichtigt (vgl. Kuhn et al. 2018).

Trotz des vermehrten Angebots an digitalen Wahlfächern findet ein Übergang in das Regelstudium für alle Medizinstudierenden nur an wenigen Universitäten statt. In Mainz und Witten/Herdecke beispielsweise ist dieser Sprung geschafft. Im Curriculum Medizin im digitalen Zeitalter erlernen die Studierenden Wissen, Fertigkeiten und Haltung zum Thema digitale Transformation und können somit digitale Kompetenzen erwerben (Kuhn und Jungmann 2018). Die Studierenden probieren selbst Gesundheits-Apps und Videosprechstunden aus. Am Schluss werden die Erfahrungen reflektiert und besprochen, um eine verantwortungsbewusste Haltung gegenüber Neuem zu erlernen. Die Reflexion sowie die ethischen Fragestellungen, die sich aus der Veränderung der Prozesse ergeben, sind als essenziell für die spätere Nutzung zu bewerten (Becka et al. 2017).

Darüber hinaus wird es künftig Modelle bzw. Studiengänge geben, die noch weitergehen. So startet im Wintersemester 2018/2019 ein erster digitaler Medizin-Studiengang nach europäischem Recht. Das EU-Studienmodell EDU (European Digital University) für Humanmedizin bietet einen dreijährigen Bachelor- und einen zweijährigen Master-ofMedicine-Studiengang an. Insgesamt umfasst die Ausbildung mindestens 5.500 Stunden Theorie und Praxis. Erster klinischer Partner für den Erwerb des Bachelors of Medicine ist die Helios Kliniken $\mathrm{GmbH}$. Es ist davon auszugehen, dass weitere Krankenhäuser folgen sollen (Ärztezeitung 2018).

\subsection{Veränderungen des Berufsbildes Arztes und dessen Spezialisierung}

In Deutschland gibt es rund 80 „spezialisierte Arztgruppen“ (Fachärzte). Es stellt sich die Frage, ob es in Zukunft noch weitere Spezialisierungen geben wird oder gar völlig neue Berufsbilder. Dies kann als mögliche Antwort auf den Fachkräftemangel in weiteren Gesundheitsfachberufen betrachtet werden und sollte immer im Zusammenspiel mit diesen bedacht werden: Immer wenn sich Disziplinen spezialisieren, droht die Gefahr, das "große Ganze“ und damit die „Heilkunst als solche“ in Frage zu stellen. Ist dem so? Zunächst ist davon auszugehen, dass Ärzte in Zukunft viel stärker interdisziplinär und übergreifend arbeiten werden. Digitalisierung schafft die Möglichkeit, dass arbeitsteilig erhobene Daten zusammengeführt werden und für eine ganzheitliche Versorgung zur Verfügung stehen. Zudem kann der interprofessionelle Diskurs durch gemeinsame Fallbesprechungen online unterstützt werden (Rebscher 2018). In Halle wurde bereits seit 2014 die Lehre interprofessionell implementiert und jetzt durch digitale Möglichkeiten erweitert. Medizinstudierende, Studierende der evidenzbasierten Pflege sowie Auszubildende der Pflege lernen in einem 
Skills Lab, das aus Simulationszentren, Future Care Labs sowie hausärztlichen Übungspraxen besteht. Hier werden neben der Lehre mit Simulationen interprofessionelle Module zur digitalen Versorgung und $\mathrm{zu}$ technischen Assistenzsystemen angeboten. Damit geht das Angebot weit über das normale Gebotene an anderen Universitätskliniken hinaus, wo in den Skills Labs die „Basisfähigkeiten“ für Medizinstudierende vermittelt werden. Die Integrationsmöglichkeiten von Emotionsrobotern in pflegerische oder therapeutische Prozesse werden ausgelotet oder der Einsatz von Kommunikationsrobotern z. B. im Vorfeld einer MRT-Untersuchung kritisch reflektiert. Im transformativen Sinn setzen sich Studierende im interprofessionellen Team mit der Technik und den kommenden Integrationsmöglichkeiten auseinander.

Diagnostische Fächer werden aller Voraussicht nach immer stärker zusammenrücken. Manche diagnostischen Disziplinen dürften sogar in einem oder zwei Gebieten aufgehen. Bildgebung, Genomdiagnostik, Pathologie, Mikrobiologie münden in Datenanalysen, die übergreifend interpretiert werden müssen. Die Entwicklung verdeutlicht den datenanalytischen Schwerpunkt, wie er als Zusatzwissen in die Diagnostikfächer einziehen wird.

Auch die gemeinnützige Stiftung Gesundheit geht davon aus, dass Ärzte zukünftig viel stärker eine Schnittstellen- und Vermittlungsfunktion wahrnehmen werden, um ein Bindeglied zwischen dem Patienten und der Maschine darzustellen und die Ergebnisse der Datenanalyse zu übersetzen (vgl. Stiftung Gesundheit 2016). „Der Arzt ist Dirigent eines großen Orchesters, idealerweise besetzt mit vielen hervorragenden Musikern" (Hofmeister 2018). Seit ein paar Jahren werden neue Berufsperspektiven diskutiert, die auch die Rolle des Arztes verändern würden. Hierzu zählen bspw. Assistenzberufe wie der Physician Assistant, der eine Art Delegationsberuf des Arztes darstellt. Oder Healthcare Content Manager, deren Aufgabe es nicht primär ist, jegliche Software für das Krankenhaus selbst zu programmieren, sondern vielmehr die richtigen Produkte am Markt zu lizensieren und die Datenhaltung im Krankenhaus bestmöglich auszugestalten (vgl. bspw. Geue et al. 2018). Zudem stellt sich die Frage, inwiefern die Akademisierung der Pflege (bspw. Advanced Nurse Practitioners bezogen auf die Rolle des Arztes als General Practitioners) die Aufgaben des Arztes tangieren wird.

Da das Wissen innerhalb der Medizin inzwischen so umfangreich und für Ärzte nur noch schwer überschaubar ist, kann es durch die Vermeidung von technischer Unterstützung zu Fehlern und einer unzureichenden Informationsverarbeitung kommen, die den Patienten am Ende sogar schaden kann (vgl. Kuhn 2018). Insofern sind technische Unterstützungssysteme auch innerhalb der Medizin nicht wegzudenken. Es stellt sich nicht mehr die Frage, ob die Digitalisierung innerhalb des Krankenhauswesens sinnvoll ist. Vielmehr sollten Ärzte Maßnahmen ergreifen, um den Prozess des digitalen Fortschritts mitzugestalten. Das bedeutet auch, dass Ärzte wieder stärker in die Verantwortung genommen werden, ihr Arbeitsumfeld selbst mit zu gestalten (vgl. Hoffmann 2018). Die Präzision und Zuverlässigkeit, die spezielle Computersysteme und Software bieten, übertrifft die Leistung des Menschen (vgl. Krüger 2018). Der Vorteil, den künstlich intelligente Systeme bieten, liegt vor allem darin, dass Maschinen „exakter und effizienter als der menschliche Verstand" arbeiten. Durch diese Präzision kann eine Sicherheit gewährleistet werden, die vor allem im Gesundheitswesen von hoher Bedeutung ist (vgl. Matusiewicz und De Witte 2018). Der Einsatz von Technologie geht vor diesem Hintergrund vor allem innerhalb der Medizin mit einem riesigen Potenzial einher (Fuchs 2016). Es ist davon auszugehen, dass der Beruf des Arztes im digitalen Zeitalter zwar nicht vollständig durch Computer und Maschinen ersetzt wird und er nicht der Arbeitslosigkeit zum Opfer fällt, aber technische Möglichkeiten zunehmend unterstützend eingesetzt und das Berufsbild des Arztes verändern werden. Hier sehen Kritiker wesentliche professionspolitische und sozialethische Herausforderungen. Die sind allerdings noch nicht dadurch gelöst, dass gezeigt werden kann, dass maschinenunterstützte Entscheidungen in definierter Weise „besser" sind als Entscheidungen von Ärzten oder interprofessionalen Teams. 


\subsection{Veränderung der Arzt-Patienten- Beziehung}

Die wesentliche Voraussetzung für eine erfolgreiche medizinische Behandlung ist die Kommunikation zwischen dem Arzt und Patienten (vgl. Meier et al. 2018). Hier herrscht im Gesundheitswesen nach wie vor eine zweiseitige Informationsasymmetrie vor, denn auch der Patient hat Informationen, die der Arzt nicht hat wie bspw. über seine Compliance (Matusiewicz et al. 2013). Da der Arzt heutzutage nicht mehr die „einzig[e] zuverlässige Quelle für gesundheitsbezogene Informationen" (VovkDebryckyi 2014) ist, verändert sich der Informationsaustausch zwischen dem Arzt und den Patienten im Zuge des digitalen Zeitalters. Davon ist auch die Ärzteschaft überzeugt: Laut einer Umfrage der Stiftung Gesundheit gehen 72 Prozent der befragten Ärzte davon aus, dass die Digitalisierung ihre Arbeit in den nächsten Jahren grundlegend verändern wird (vgl. Stiftung Gesundheit 2017). Da der bisherige Wissensvorsprung des Arztes zunehmend zu schwinden scheint, wird der gut informierte Patient in Zukunft vor allem einen Arzt an seiner Seite brauchen, der ihm Orientierung bietet. Viele Patienten informieren sich heute schon über das Internet über ihre vorhandenen Beschwerden und mögliche Krankheiten. Zusätzliche Anamnese-Apps bieten dem Patienten eine Voreinschätzung und gegebenenfalls dem Arzt eine Hilfestellung, um bei einer krankheitsspezifischen Anamnese nichts zu vergessen. So unterstützen digitale Prozesse die gemeinsame Entscheidungsfindung und ermöglichen so eine stärkere Einbindung des Patienten in den Behandlungsprozess. Der digitale und emanzipierte Patient, der sich über die Diagnose, den Therapieverlauf oder den Behandlungsprozess informiert, möchte mitreden und in Entscheidungsprozesse eingebunden werden.

Es gibt aber auch neue Herausforderungen. Laut einer Umfrage der Bertelsmann Stiftung empfindet fast jeder zweite Arzt diejenigen Patienten, die sich im Internet über ihre Symptome informieren, als störend (Liebrich 2017). Denn oftmals kommt es unnötig zu falschen Annahmen und Fehlinterpretationen, in die sich Patienten hineinsteigern. Vor diesem Hintergrund ist in den vergangenen Jahren der Begriff der Cyberchondrie oder Googlechond- rie entstanden (Peikert 2016). Die Informationsflut an medizinischem Wissen und die Missverständnisse verdeutlichen, wie wichtig die Rolle des Arztes ist. Dieser kann Aufklärungsarbeit leisten, Fehlinterpretationen und Missverständnisse vermeiden und Fragen beantworten. $\mathrm{Zu}$ einem ähnlichen Ergebnis kommt eine Umfrage des Digitalverbandes Bitkom und des Ärzteverbandes Hartmannbund: 64 Prozent der befragten Ärzte gaben an, dass der Umgang mit Patienten, die sich vorab im Internet informieren, anstrengend ist. Allerdings gaben 51 Prozent der Befragten an, dass sie vom gut informierten Patienten schon profitieren konnten (Bitkom Research 2017). Dies zeigt, dass die bisherigen Erfahrungen mit dem Einsatz von technischen und digitalen Lösungen ambivalent bewertet werden. Empirische Studien der Universität Witten/ Herdecke und der Harvard University in Boston belegen, dass sich das Verhältnis zwischen dem Arzt und den Patienten verbessert, wenn die Patienten Einblick in die eigenen medizinischen Befunde und ärztliche Notizen erhalten (Esch et al. 2016). Durch die Transparenz kann der Patient die Diagnose besser nachvollziehen und es kann eine vertrauensvolle Beziehung zwischen Arzt und Patient aufgebaut werden. Ein transparenter Umgang mit den eigenen Daten kann den diagnostischen Verlauf und Behandlungsprozess positiv beeinflussen (vgl. Liebrich 2017). Der persönliche Austausch zwischen dem Arzt und den Patienten ist vor dem Hintergrund eines zunehmend uneingeschränkten $\mathrm{Zu}$ griffs auf Informationen und Daten einerseits, aber auch im Sinne der Komplexitätsreduktion andererseits für die Vermeidung von Fehlinterpretationen und Missverständnissen unabdingbar. Der Arzt wird dem Patienten im Zeitalter des digitalen Wandels zunehmend beratend zur Seite stehen und verstärkt eine Schnittstellen- und Vermittlungsfunktion wahrnehmen. Vor allem die Kommunikationsfähigkeit von Ärzten und die Fähigkeit zur Empathie stellen Kompetenzen dar, auf die es in Zukunft bei Ärzten ankommen wird. Der Abschied vom Paternalismus in der Medizin ist nicht erst Folge der Digitalisierung, aber diese trägt maßgeblich dazu bei. Der Arzt muss sich viel stärker auf die individuellen Wünsche und Bedürfnisse des $\mathrm{Pa}$ tienten einlassen und diesen in den Behandlungsprozess einbinden. Das setzt voraus, dass der Arzt 
gut zuhören kann und verständlich kommuniziert. Da die Medizin immer spezieller und komplexer wird, muss der Arzt in der Lage sein, diese Information verständlich $\mathrm{zu}$ vermitteln. Eine weitere wichtige Fähigkeit, die im ärztlichen Berufsalltag noch bedeutsamer sein wird, ist das Einfühlungsvermögen: Empathie ist eine grundlegende Voraussetzung des Arztes, um den Patienten zu seinen Symptomen zu befragen, sich in die Lage des $\mathrm{Pa}$ tienten hineinzuversetzen und eine kontextspezifische Diagnose aufzustellen. Es gibt allerdings auch kritische Diskussionen, ob dieses „sich hineinversetzen“ nicht bloß die Rückkehr eines unreflektierten Paternalismus ist (Stanghellini und Rosfort 2013; Bloom 2016; Ariso 2018). Empathie muss und kann man auch lernen. Die Fähigkeit zur Empathie unterscheidet Ärzte von Maschinen. Vor diesem Hintergrund spielt die Empathie des Arztes, die auch bedeutsam für den Heilungsprozess ist, auch im Zeitalter der Digitalisierung eine wichtige Rolle. Aus Sicht der Patienten zählen Empathie, eine verständliche Kommunikation und die Wahrung der Autonomie des Patienten zu den Aspekten, die sie vom Arzt erwarten (vgl. Hofmann 2018). Zusammenfassend wird der persönliche Austausch zwischen dem Arzt und den Patienten auch in Zeiten des digitalen Wandels von grundlegender Bedeutung für eine erfolgreiche Diagnose und passgenaue Therapie bleiben und vielleicht sogar noch verstärkter eingefordert (vgl. Meier et al. 2018).

\subsection{Fazit}

Die digitale Transformation des Krankenhauses wird die Berufsausübung von Ärzten zunehmend beeinflussen. Der punktuelle Einsatz von intelligenten digitalen Systemen im Krankenhaus zeigt heute schon, dass der Wandel mit neuen beruflichen Anforderungen an die Ärzte verbunden ist. Hierdurch wird allerdings nicht immer nur das Arzt-Patienten-Verhältnis tangiert. In der Zahnmedizin sind z. B. digitale Volumentomographie und digitale Werkstückbearbeitung verfügbar und haben die Arbeitsteilung zwischen Zahnarzt und Zahntechniker geändert. Zudem ist davon auszugehen, dass sich das Berufsbild des Arztes weiter spezialisieren wird und neue Assistenzberufe entstehen werden. Spezi- alisierung bedeutet nicht eine Zunahme medizinischer Fächer, vielmehr werden beispielsweise die Grenzen zwischen diagnostischen Disziplinen durchlässiger, interdisziplinäre Datenanalysen rücken in den Vordergrund. Ärzte werden in Zukunft weiter gebraucht - allerdings in einer anderen Rolle. Während die Maschine stark in der Diagnostik unterstützen wird, wird der Arzt in der Interpretation und Kommunikation der Ergebnisse stärker gefragt sein.

Wie empirische Studien zeigen, bewertet die Ärzteschaft diesen Veränderungsprozess in Bezug auf ihr eigenes Berufsbild ambivalent. Einerseits steht sie den neuen technischen Errungenschaften positiv gegenüber, da diese eine Möglichkeit bieten, die Krankenhausprozesse z. B. in Bezug auf die zunehmende Bürokratisierung zu optimieren und die medizinische Versorgungsqualität auch im Zuge des demografischen Wandels sicherzustellen. Andererseits behindern Bedenken in Bezug auf die ITSicherheit und die strengen datenschutzrechtlichen Vorgaben bislang die flächendeckende Einführung von digitalen Lösungen im Gesundheitswesen.

Dies spiegelt sich in einer zu verändernden Medizinerausbildung wider, bei der auch eine digitale Kompetenz an Bedeutung gewinnen wird. Die Digitalisierung der medizinischen Ausbildung hat an den verschiedenen Standorten in Deutschland unterschiedlich schnell Einzug gefunden. Einige Leuchtturm-Projekte machen die Beschäftigung mit dem Thema bereits jetzt im Medizinstudium möglich. Gerade dadurch, dass dieser Bereich in der medizinischen Ausbildung sehr neu ist, können neue Konzepte ausprobiert und integriert werden. Des Weiteren sind noch mehr neue Konzepte denkbar, die auch gerade in der medizinischen Weiterbildung Einzug finden können. Es werden neue Fragen aufgeworfen, denen die medizinische Ausbildung sich jetzt stellen sollte. Sind die Kompetenzen, die jetzt erlernt werden, schon wieder überholt, wenn die Ausbildung fertig ist? Sollte es in Zukunft vielleicht verpflichtend werden, Operationen in Simulationen zu üben, bevor sie am richtigen $\mathrm{Pa}$ tienten durchgeführt werden? Für welche Diagnose und Therapieschritte brauchen wir noch mehr digitalen Wandel? Da neue Anforderungen an die Lehrenden über alle Berufs- und Fachgruppen hinweg gestellt werden, besteht zudem eine Chance für eine 
verstärkte Zusammenarbeit der Berufsgruppen im Gesundheitswesen.

Zudem ist in vielen Branchen eine Konzentration zu Plattform- und Netzwerkökonomien erkennbar. Das Krankenhaus wird zunehmend im internationalen Wettbewerb stehen und mit bspw. der Mayo Clinic in den USA im Bereich der Diagnostik konkurrieren. Auch der Arztberuf wird daher ein Stück weit internationaler werden.

Die digitale Transformation...

- wird den Arztberuf grundlegend verändern, ohne ihn zu ersetzen,

- teilt die Ärzteschaft in Befürworter und Kritiker,

- wird die Ausbildung der Mediziner maßgeblich verändern und hebt die Qualifikationsanforderungen unter den Medizinstudierenden,

- verstärkt die interprofessionelle Zusammenarbeit zwischen den unterschiedlichen Berufsgruppen,

- wird im Zuge der Plattform- und Netzwerkökonomien das Krankenhaus unter internationalen Wettbewerb stellen.

\section{Literatur}

Altpeter (2017) E-Health als Bestandteil ganzheitlicher Therapieoptimierung. Diabetologe:13:29-37

Ariso JM (2018) Enhancing second-order empathy in medical practice by supplementing patients' narratives with certainties. BMC Med Educ 18(1):35

Ärztezeitung (2018) Privatuni bietet EU-Studienmodell Humanmedizin. https://www.aerztezeitung.de/praxis_ wirtschaft/ausbildung/article/967359/ausbildung-privatuni-bietet-eu-studienmodell-humanmedizin.html

Bauer M (2017) So verändert Frauenpower die Digitalisierung im Gesundheitswesen. Healthcare Startups Deutschland. https://healthcare-startups.de/so-veraendert-frauenpower-die-digitalisierung-im-gesundheitswesen/. Zugegriffen: 14. Juni 2018

Becka D, Evans M, Hilbert J (2017) Digitalisierung in der sozialen Dienstleistung. FGW-Studie Digitalisierung von Arbeit. ISSN 2510-4010

Bitkom Research (2017) Gesundheit 4.0 - Wie Ärzte die digitale Zukunft sehen. https://www.hartmannbund.de/ fileadmin/user_upload/Downloads/Umfragen/2017_ HB-Bitkom_Start-ups.pdf. Zugegriffen: 14. Juni 2018

Bloom P (2016) Against empathy: The case for rational compassion, first edition. Ecco, New York

BMBF (2017) Masterplan Beschlusstext. https://www.bmbf.de/ files/2017-03-31_Masterplan\%20Beschlusstext.pdf
Boeker M, Klar R (2006) E-Learning in der ärztlichen Aus- und Weiterbildung. Methoden, Ergebnisse, Evaluation. Bundesgesundheitsblatt Gesundheitsforschung Gesundheitsschutz 49(5):405-411

Bundesärztekammer (2017) 120. Deutscher Ärztetag 2017 in Freiburg. http://www.bundesaerztekammer.de/aerztetag/aerztetage-ab-2006/120-deutscher-aerztetag2017-in-freiburg/. Zugegriffen: 18 Jun 2018

Ellaway R, Poulton T, Fors U, McGee JB, Albright S (2008) Building a Virtual Patient Commons. Medical Teacher 30(2):170-74. https://doi. org/10.1080/01421590701874074

Esch T, Mejilla R, Anselmo M, Podtschaske B, Delbanco T, Walker J (2016) Engaging patients through open notes: an evaluation using mixed methods. BMJ open 6(1), e010034

Feldmann J, Wolf D (2016) Hochschule 4.0. In: Wolff D, Göbel R (Hrsg) Digitalisierung: Segen oder Fluch? Springer Nature, Heidelberg, S 191-223

Fuchs M (2016) Der digitale Doktor. Frankfurter Allgemeine Sonntagszeitung. http://www.faz.net/aktuell/berufchance/beruf/koennen-computer-aerzte-anwaelte-und-wirtschaftspruefer-ersetzen-14282103.html. Zugegriffen: 16. Juni 2018

Geue D, Schreiber A, Lerner M (2018) Healthcare Content Marketing für Dummies, 1. Aufl. Wiley, Weinheim

Henn H (2015) Landarzt Dr. Google: Wie die Digitalisierung das Gesundheitswesen revolutioniert. https://www. smarter-service.com/2015/09/14/landarzt-dr-google-wie-die-digitalisierung-das-gesundheitswesen-revolutioniert/. Zugegriffen: 14. Juni 2018

Hochschulforum Digitalisierung (2016) The Digital Turn Hochschulbildung im digitalen Zeitalter. Arbeitspapier Nr. 27. Hochschulforum Digitalisierung, Berlin

Hofmann M (2018) Angehende Ärzte sollen zu Menschen werden, die bereit sind, das System zu verändern. https:// drnext.de/interview-marzellus-hofmann/. Zugegriffen: 14. Juni 2018

Hofmeister S (2018) Wir brauchen den Arzt künftig als Schnittstelle zwischen Mensch und Maschine. Stephan Hofmeister im Interview mit Dr. Next. https://drnext.de/ interview-stephan-hofmeister/. Zugegriffen: 14. Juni 2018

Horak P et al (2017) Precision oncology based on omics data: the NCT Heidelberg experience. International Journal of Cancer 141(5):877-886

Kade-Lamprecht E, Sander M (2017) Was kann die Gesundheitswirtschaft aus dem Retail lernen? In: Matusiewicz D, Muhrer-Schwaiger M (Hrsg) Neuvermessung der Gesundheitswirtschaft. Springer Gabler, Wiesbaden, S 145-158

Keller N, Jenny MA, Gigerenzer G, Ablin RJ (2018) PSA-Screening: Möglicher Nutzen und Schaden. Deutsches Ärzteblatt 115(13):A-583

Krüger M (2018) Was macht die Digitalisierung mit der Attraktivität des Arztberufes? Passion Chirurgie 03/2018. https://www.bdc.de/was-macht-die-digitalisierung-mit-der-attraktivitaet-des-arztberufes/. Zugegriffen: 12. Juni 2018 
Kuhn S, Jungmann F (2018) Medizin im digitalen Zeitalter. Der Radiologe 58 (3):236-240 https://doi.org/10.1007/ s00117-017-0351-7

Kuhn S, Frankenhauser S, Tolks D (2018) Digitale Lehr- und Lernangebote in der medizinischen Ausbildung: Schon am Ziel oder noch am Anfang? Bundesgesundheitsblatt Gesundheitsforschung Gesundheitsschutz 61(2):201-209

Kuhn S et al (2017) Medizin im digitalen Zeitalter: Was bedeutet das für die Ausbildung im Medizinstudium? Arbeitsmed Sozialmed Umweltmed 52(6):418-422

Kuhn S, Frankenhauser S, Tolks D (2018) Digitale Lehr- und Lernangebote in der medizinischen Ausbildung. Springer. EJ853864.pdf. n.d. https://files.eric.ed.gov/ fulltext/EJ853864.pdf. Zugegriffen: 08. Juli 2018

Kuhn S, Kadioglu D, Deutsch K, Michl S (2018) Data Literacy in der Medizin. Der Onkologe 24(5):368-377 https://doi. org/10.1007/s00761-018-0344-9

Lage MJ, Platt GJ, Treglia M (2000) Inverting the Classroom: A Gateway to Creating an Inclusive Learning Environment. The Journal of Economic Education 31 (1):30-43. DOI: 10.1080/00220480009596759

Lang S (2017) Finanzielle und berufspolitische Fallstricke der Telemedizin müssen trotz guter Akzeptanz unter Patienten mitberücksichtigt werden. Karger Kompass Pneumol 5:28-29

Liebrich F (2017) Digitale Medienprodukte in der ArztPatienten-Kommunikation. Chancen und Risiken einer personalisierten Medizin. Springer Vieweg, Wiesbaden

Marburger Bund (2017) MB-Monitor 2017. Zu wenig Zeit für Patienten, zu viel Arbeit mit Bürokratie. http://www. marburger-bund.de/der-marburger-bund/projekte-und-kampagnen/mitgliederbefragung/2017. Zugegriffen: 22. Juli 2018

Matusiewicz D, De Witte B (2018) Risikofaktor Mensch Ist die Maschine der bessere Arzt? In: Oubaid V. RISIKO MENSCH! Der Humanfaktor in der Patientensicherheit. MWV-Verlag, Berlin

Matusiewicz D, Wasem J, Lux G (2013) Patienteninformation und -kommunikation im Versorgungsmanagement eine kritische Abhandlung. In: Burger S (Hrsg) Alter und Multimorbidität - Herausforderungen an die Gesundheitsversorgung und die Arbeitswelt. medhochzwei, Heidelberg, S 207-226

Matusiewicz D, Pittelkau C, Elmer A (2017) Die Digitale Transformation im Gesundheitswesen, 1. Aufl. MWV-Verlag, Berlin

Meier R, Holderried M, Kraus, Tobias M (2018) Digitalisierung der Arzt-Patienten-Kommunikation. In: Pfannstiel MA, Da-Cruz P, Rasche C (Hrsg) Entrepreneurship im Gesundheitswesen III. Digitalisierung - Innovationen - Gesundheitsversorgung. Springer Gabler, Wiesbaden, S 63-77

MFT Medizinischer Fakultätentag der Bundesrepublik Deutschland e. V. (2015) Nationaler Kompetenzbasierter Lernzielkatalog. http://www.nklm.de/files/nklm_final_2015-07-03.pdf
Michel A, Baumgartner P, Brei C, Hesse F, Kuhn S, Pohlenz P, Quade S, Seidl T, Spinath B (2018) Framework zur Entwicklung von Curricula im Zeitalter der digitalen Transformation. Diskussionspapier Nr. 01. Berlin: Hochschulforum Digitalisierung

Nelles (2017) Teleneurologie - Chancen und Risiken. Nervenarzt 88:328-342

Peikert D (2016) Plötzlich hat jeder Krebs. Frankfurter Allgemeine Zeitung. http://www.faz.net/aktuell/gesellschaft/ gesundheit/aerzte-kritisieren-internetrecherche-vonpatienten-14294975.html. Zugegriffen: 22. Juni 2018

Persike M, Friedrich J (2016) Lernen mit digitalen Medien aus Studierendenperspektive. http://www.che-ranking.de/ downloads/HFD_AP_Nr_17_Lernen_mit_digitalen_ Medien_aus_Studierendenperspektive.pdf

PWC (2016) Strategy\&. Weiterentwicklung der eHealthStrategie: Studie im Auftrag des Bundesministeriums für Gesundheit. Berlin. https://www.bundesgesundheitsministerium.de/fileadmin/Dateien/3_Downloads/E/ eHealth/BMG-Weiterentwicklung_der_eHealth-Strategie-Abschlussfassung.pdf. Zugegriffen: 14. Juni 2018

Rebscher H (2018) Gesundheitsökonomie vor alten und neuen Herausforderungen: ordnungsökonomische Fundierung nötiger denn je. Gesundh ökon Qual manag 23:109-110

Schaeffer D et al (2018) Der Nationale Aktionsplan Gesundheitskompetenz - Relevanz für die Versorgungsforschung. Monitor Versorgungsforschung, Ausgabe 4/2018, S 53-58

Stanghellini G, Rosfort R (2013) Empathy as a sense of autonomy. Psychopathology 46(5):337-344

Stegmann K, Fischer F (2016) Auswirkungen digitaler Medien auf den Wissens- und Kompetenzerwerb an der Hochschule. Forschungsbericht. https://epub.ub.unimuenchen.de/38264/

Stiftung Gesundheit (2017) Ärzte im Zukunftsmarkt Gesundheit 2017: Transsektorale Zusammenarbeit. Stiftung Gesundheit (Hrsg). https://www.stiftung-gesundheit.de/ pdf/studien/Aerzte_im_Zukunftsmarkt_Gesundheit_2017.pdf. Zugegriffen: 20. Juni 2018

von Hirschhausen E (2018) Für den Arzt der Zukunft fehlen offensichtlich Rollenmodelle, Vorbilder sind rar. Eckart von Hirschhausen im Interview mit Dr. Next. https:// drnext.de/interview-eckart-von-hirschhausen/. Zugegriffen: 20. Juni 2018

Vovk-Debryckyi O (2014) Die zeitgemäße Arzt-Patient-Beziehung. Was erwarten sich Patienten vom Besuch in der Praxis? Dissertation. Ludwig-Maximilians-Universität, München

Wissenschaftsrat (2014) Empfehlungen zur Weiterentwicklung des Medizinstudiums in Deutschland auf Grundlage einer Bestandsaufnahme der humanmedizinischen Modellstudiengänge. https://www.wissenschaftsrat.de/ download/archiv/4017-14.pdf 
Open Access Dieses Kapitel wird unter der Creative Commons Namensnennung 4.0 International Lizenz(http://creativecommons. org/licenses/by/4.0/deed.de) veröffentlicht, welche die Nutzung, Vervielfältigung, Bearbeitung, Verbreitung und Wiedergabe in jeglichem Medium und Format erlaubt, sofern Sie den/die ursprünglichen Autor(en) und die Quelle ordnungsgemäß nennen, einen Link zur Creative Commons Lizenz beifügen und angeben, ob Änderungen vorgenommen wurden.

Die in diesem Kapitel enthaltenen Bilder und sonstiges Drittmaterial unterliegen ebenfalls der genannten Creative Commons Lizenz, sofern sich aus der Abbildungslegende nichts anderes ergibt. Sofern das betreffende Material nicht unter der genannten Creative Commons Lizenz steht und die betreffende Handlung nicht nach gesetzlichen Vorschriften erlaubt ist, ist für die oben aufgeführten Weiterverwendungen des Materials die Einwilligung des jeweiligen Rechteinhabers einzuholen. 